\title{
Profound vascular stasis of retina and optic nerve following retrobulbar anesthesia
}

\author{
This article was published in the following Dove Press journal: \\ International Medical Case Reports Journal \\ 29 April 2016 \\ Number of times this article has been viewed
}

\author{
Bozho Todorich ${ }^{1,2}$ \\ Paul Hahn² \\ 'Associated Retinal Consultants and \\ Oakland University William Beaumont \\ School of Medicine, Royal Oak, MI, \\ ${ }^{2}$ Duke Eye Center, Duke University \\ Medical Center, Durham, NC, USA
}

Introduction: We aim to describe a mechanism of vision loss following vitrectomy surgery with retrobulbar block (RBB) associated with severe vascular stasis of the optic nerve and macula in order to improve safety of local anesthesia for ophthalmic surgery.

Case presentation: We report three cases of patients who underwent pars plana vitrectomy (PPV) with retrobulbar anesthesia with no retrobulbar hemorrhage or elevated intraocular pressure (IOP). At the beginning, in each case, hypoperfusion of optic nerve and macula was noted. In the case of one patient with significant vasculopathic risk factors, the vascular stasis was severe, while in the other two cases, it was mild-to-moderate. In all cases, the perfusion of posterior pole began to improve almost immediately following the start of PPV. Because the IOP was not elevated and no retrobulbar hemorrhage was present, this suggested a compartment syndrome in the intraconal space. The patient with severe vascular stasis developed finger-counting vision but had normal postoperative angiogram findings and unrevealing cardiovascular workup. In the other two milder cases, the occurrence of ischemia was not visually significant.

Conclusion: Intraoperative ischemia should be considered in all cases of unexplained vision loss after ophthalmic surgery using RBB. Attention to vasculopathic risk factors and intraoperative hemodynamic parameters, in addition to the use of parabulbar block, may avoid this complication and permanent vision loss.

Keywords: macular ischemia, pars plana vitrectomy, retrobulbar block, central retinal artery occlusion

\section{Introduction}

Retrobulbar blocks (RBBs) are commonly used for anesthesia and akinesia during ophthalmic procedures. Known complications of RBB include retrobulbar hemorrhage, inadvertent globe perforation, central nervous system toxicity, and rarely optic neuropathies. ${ }^{1}$ Retrobulbar hemorrhage occurs in $0.1 \%-1.7 \%$ of cases of RBB, and its risk is associated with advanced age, systemic anticoagulants, nonsteroidal antiinflammatory drugs, and steroids. Increased intraconal pressure results in increased intraocular pressure (IOP), which can impede perfusion of optic nerve and central retinal artery. ${ }^{2}$ Although prompt decompression is usually successful, patients may develop persistent scotoma or optic neuropathy.

Although extremely rare, retinal vascular events, such as central retinal artery and vein occlusions, have been described in association with RBB in the absence of associated retrobulbar hemorrhage. ${ }^{3-5}$ In contrast to other intraocular procedures, pars plana vitrectomy (PPV) surgery affords a unique opportunity to visualize the retinal vasculature immediately following RBB. We report three cases of severe hypoperfusion and vascular
Correspondence: Paul Hahn

Duke Eye Center, Duke University Medical Center, Box 3802, 235 I Erwin

Road, Durham, NC 277I0, USA

Email paulhahn@gmail.com
International Medical Case Reports Journal 2016:9 109-II2

109

Dovepress

http://dx.doi.org/10.2147/IMCRJ.S98546 (c) (1) (5) 2016 Todorich and Hahn. This work is published and licensed by Dove Medical Press Limited. The full terms of this license are available at https://www.dovepress. com/ cc. work you hereby accept the Terms. Non-commercial uses of the work are permitted without any further permission from Dove Medical Press Limited, provided the work is properly attributed. For permission for commercial use of this work, please see paragraphs 4.2 and 5 of our Terms (https://www.dovepress.com/terms.php). 
stasis in the macula at the beginning of vitrectomy surgery after RBB, which, in one case, became visually significant.

\section{Case presentation}

As the surgery was performed as standard of care, but not part of experimental protocol, the following study was exempt from institutional review board approval, but informed consent was obtained from the patients. Three patients underwent PPV for recurrent macula-on retinal detachment, full-thickness macular hole, and epiretinal membrane (Table 1). All three patients received RBB preoperatively in standard fashion with an Atkinson $38 \mathrm{~mm}$ retrobulbar needle advanced into the intraconal space through the inferotemporal quadrant. Approximately 5-7 mL of anesthetic (lidocaine and bupivacaine; Table 1) was administered following plunger pullback to verify that an orbital vessel was not cannulated. The eye was manually massaged post-RBB, and there was no evidence of retrobulbar hemorrhage, elevated IOP by palpation, or other complications following administration.

A standard three-port PPV setup (23 G or $25 \mathrm{G}$ ) with valved cannulas was used. At the beginning of each procedure, optic nerve pallor, hypoperfusion of the optic nerve head, retinal vascular stasis as well as attenuation and box-carring of the retinal vessels were noted (Figure 1A). After engaging in aspiration-cutting for core vitrectomy, improvement in perfusion of the optic nerve and the macula was noted almost immediately (Figure 1B). In two cases, the vascular stasis was subjectively mild-to-moderate at the beginning of the case, involving smaller vessels without significant optic nerve pallor and this normalized immediately with start of vitrectomy. In one case, the vascular stasis was subjectively severe at the beginning (Figure 1), involving all arcade vessels with marked optic nerve pallor. Perfusion only slowly returned following initiation of core vitrectomy and throughout the course of the subsequent stages of the surgery, including membrane staining and peeling (Figure 1C and D). At the conclusion of the case, perfusion was noted to normalize, with resolution of visible optic nerve pallor (Figure 1D). Postoperative fluorescein angiography and clinical examination did not reveal perfusion abnormalities in any case. The postoperative optical coherence tomography demonstrated successful membrane peel (Figure 1E and F), with no significant alteration in retinal anatomy that would account for the severity of visual acuity loss. A cardiovascular workup performed postoperatively on the severe case, who had a prior history of giant cell arteritis (albeit without ophthalmic involvement), hypertension, and cardiac and carotid disease, included normal resting cardiac echocardiogram and carotid Doppler ultrasound which demonstrated carotid stenosis of $<50 \%$ bilaterally with patent stents. In the two mild-to-moderate cases, these findings did not appear to significantly affect final visual outcome. In the severe case, the patient's preoperative vision of 20/200 deteriorated to "count fingers" visual acuity despite improved anatomy.

\section{Discussion}

We report three cases of vascular stasis after RBB, which improved after initiation of vitrectomy without observable postoperative perfusion abnormalities. All of these cases underwent uncomplicated administration of $\mathrm{RBB}$ without evidence of retrobulbar hemorrhage or elevated IOP by palpation. One limitation of our study is its retrospective nature, which precluded rigorous intraoperative IOP determination because tonometry is not part of surgical routine during vitrectomy at our institution. Future prospective study on this issue will clarify whether occult IOP elevation not detected by digital palpation might have contributed to the observed findings.

Table I Demographics, background patient and surgeon characteristics, past medical history, and outcomes of patients included in the series

\begin{tabular}{|c|c|c|c|}
\hline & Case I (February 7, 20I3) & Case 2 (February 28, 20I3) & Case 3 (March 26, 2013) \\
\hline Operating room & Satellite hospital & Satellite hospital & University hospital \\
\hline \multirow[t]{2}{*}{ Surgical indication } & ERM & Macula-on recurrent & FTMH \\
\hline & & $\mathrm{RD} s / \mathrm{p}$ recent $\mathrm{SB}$ & \\
\hline \multirow[t]{2}{*}{$\mathrm{PMHx}$} & History of GCA (no ophthalmic & Healthy & Healthy \\
\hline & involvement), s/p carotid EA, s/p CABG & & \\
\hline Block & RBB ( $5 \mathrm{~mL}: 2 \%$ lido [+MP], $0.75 \%$ bupi) & RBB ( $5 \mathrm{~mL}: 2 \%$ lido [+MP], $0.75 \%$ bupi) & RBB (7 mL: $4 \%$ lido [-MP], $0.75 \%$ bupi, hyal) \\
\hline Surgeon & Attending surgeon only & Attending surgeon only & Fellow/attending \\
\hline Vascular stasis & Severe & Mild (immediately improved with VTX) & Mild (immediately improved with VTX) \\
\hline Visual outcome & $\mathrm{CF}$ & $20 / 32$ & 20/50 (POM\#3, then LTF) \\
\hline
\end{tabular}

Abbreviations: ERM, epiretinal membrane; RD, retinal detachment; SB, scleral buckle; FTMH, full-thickness macular hole; GCA, giant cell arteritis; carotid EA, carotid endarterectomy; CABG, coronary artery bypass grafting; RBB, retrobulbar block; lido, lidocaine; bupi, bupivacaine; hyal, hyaluronidase; MP, methylparaben; VTX, vitrectomy; CF, count fingers; POM\#3, postoperative month 3; LTF, lost to follow-up; PMHx, past medical history. 

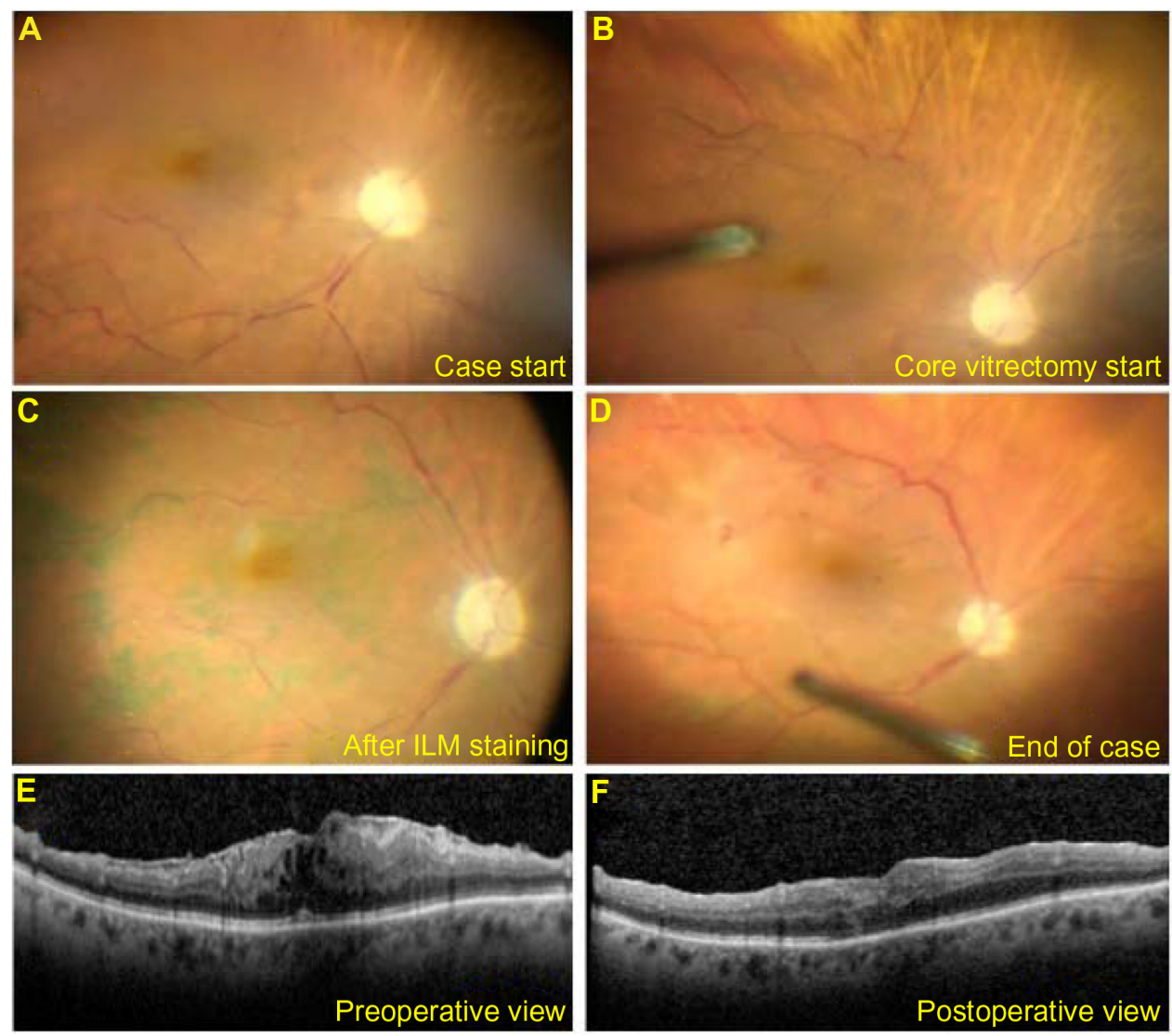

Figure I Intraoperative view of optic nerve and macular ischemia in Case I patient.

Notes: At the beginning of the case, prior to initiation of vitrectomy, marked optic nerve pallor, retinal vessel attenuation, and box-carring were noted (A). There was instantaneous but partial improvement in optic nerve and macular perfusion as soon as the vitreous cutter was engaged (B). The perfusion continued to improve throughout the procedure, as shown after ILM staining (C) and after membrane peeling (D). Macular OCT shows preoperative ERM (E). At 6-week follow-up, there is improved macular thickening and resolution of intraretinal cysts but significantly worsened visual acuity $(\mathbf{F})$.

Abbreviations: ERM, epiretinal membrane; ILM, internal limiting membrane; OCT, optical coherence tomography.

The ischemic events described in this study were noted in different hospitals with different surgeons administering the block, suggesting that this is not related to technique or idiosyncrasies in block composition. There have been reports of epinephrine-associated vasoconstriction resulting in similar findings,${ }^{6,7}$ but none of the block components in our series contained epinephrine. In a study of postvitrectomy optic neuropathy, $71 \%$ of patients experienced intraoperative hypotension, which was hypothesized to result in a drop in intraocular perfusion pressure; ${ }^{8}$ in our cases, no intraoperative drop in systemic blood pressure was documented. Similar findings have also been reported following administration of methylparaben (MP)-preserved anesthetics. ${ }^{9}$ While two of the cases were injected with MP-preserved lidocaine, the third case of this series was injected with preservative-free anesthetics.

Considering the immediate improvements following initiation of vitrectomy, which may result in an instantaneous decrease in IOP, we hypothesize that a localized compartment syndrome in the intraconal space decreases intraocular perfusion without gross elevation in IOP. In the two otherwise-healthy individuals, these findings did not appear to be visually significant. However, in one patient with numerous vasculopathic risk factors, these intraoperative findings were more severe and were associated with permanent vision loss. This suggests that underlying vascular status is important in modulating the perfusion response of the optic nerve and the macula to alterations in ocular perfusion pressure during surgery. Although intraoperative ischemia was readily apparent at the beginning of PPV as direct visualization of the posterior pole enabled its rapid recognition, this can occur in any ophthalmic surgery, such as cataract surgery, which uses RBB. Recognition of this occurrence is difficult and high index of suspicion is needed in these cases, as surgeons routinely do not use ophthalmoscopy following RBB administration.

Following these ischemic events, the authors have transitioned to the use of peribulbar, rather than retrobulbar, 
blocks and have not experienced similar findings since. At a minimum, we suggest that a peribulbar, rather than retrobulbar, block be strongly considered in patients with such vasculopathic risk factors. Conversely, using a conjunctival cutdown with administration of retrobulbar anesthesia using cannula on the field may be another alternative to a traditional RBB. Careful attention to hemodynamic factors such as intraoperative blood pressure control, in coordination with the anesthesia service, may be important to avoid hypotensive episodes that would further compromise ocular perfusion pressure.

\section{Conclusion}

Vitrectomy surgery affords an opportunity to visualize the retinal vasculature following administration of RBB and provides unique insight into the vascular mechanism governing intraoperative ocular perfusion. Beyond vitrectomy, however, the risk of visually significant intraoperative ischemia extends to any form of ophthalmic surgery using RBB. We propose that vascular changes may be involved in the etiology of otherwise-unexplained vision loss following intraocular surgery with retrobulbar anesthesia.

\section{Disclosure}

$\mathrm{BT}$ reports no conflicts of interest in this work, $\mathrm{PH}$ has a consulting agreement unrelated to the submitted work with
Second Sight Medical Products, Inc. The authors report no other conflicts of interest in this work.

\section{References}

1. Eke T, Thompson JR. The national survey of local anaesthesia for ocular surgery. II. Safety profiles of local anaesthesia techniques. Eye. 1999;13(pt 2):196-204.

2. Cionni RJ, Osher RH. Retrobulbar hemorrhage. Ophthalmology. 1991;98:1153-1155.

3. Klein ML, Jampol LM, Condon PI, Rice TA, Serjeant GR. Central retinal artery occlusion without retrobulbar hemorrhage after retrobulbar anesthesia. Am J Ophthalmol. 1982;93:573-577.

4. Sullivan KL, Brown GC, Forman AR, Sergott RC, Flanagan JC. Retrobulbar anesthesia and retinal vascular obstruction. Ophthalmology. 1983;90:373-377.

5. Vinerovsky A, Rath EZ, Rehany U, Rumelt S. Central retinal artery occlusion after peribulbar anesthesia. J Cataract Refract Surg. 2004;30:913-915.

6. Lamichhane G, Gautam P. Central retinal arterial occlusion (CRAO) after phacoemulsification-a rare complication. Nepal J Ophthalmol. 2013;5:281-283.

7. Chang HL. Temporary amaurosis following retrobulbar injection of novocain-adrenalin in glaucoma. Chin Med J. 1956;74:116-124.

8. Bansal AS, Hsu J, Garg SJ, et al. Optic neuropathy after vitrectomy for retinal detachment: clinical features and analysis of risk factors. Ophthalmology. 2012;119(11):2364-2370.

9. Tappeiner C, Garweg JG. Retinal vascular occlusion after vitrectomy with retrobulbar anesthesia-observational case series and survey of literature. Graefes Arch Clin Exp Ophthalmol. 2011;249:1831-1835.
International Medical Case Reports Journal

\section{Publish your work in this journal}

The International Medical Case Reports Journal is an international, peer-reviewed open-access journal publishing original case reports from all medical specialties. Previously unpublished medical posters are also accepted relating to any area of clinical or preclinical science. Submissions should not normally exceed 2,000 words or

\section{Dovepress}

4 published pages including figures, diagrams and references. The manuscript management system is completely online and includes a very quick and fair peer-review system, which is all easy to use. Visit $\mathrm{http}: / /$ www.dovepress.com/testimonials.php to read real quotes from published authors. 